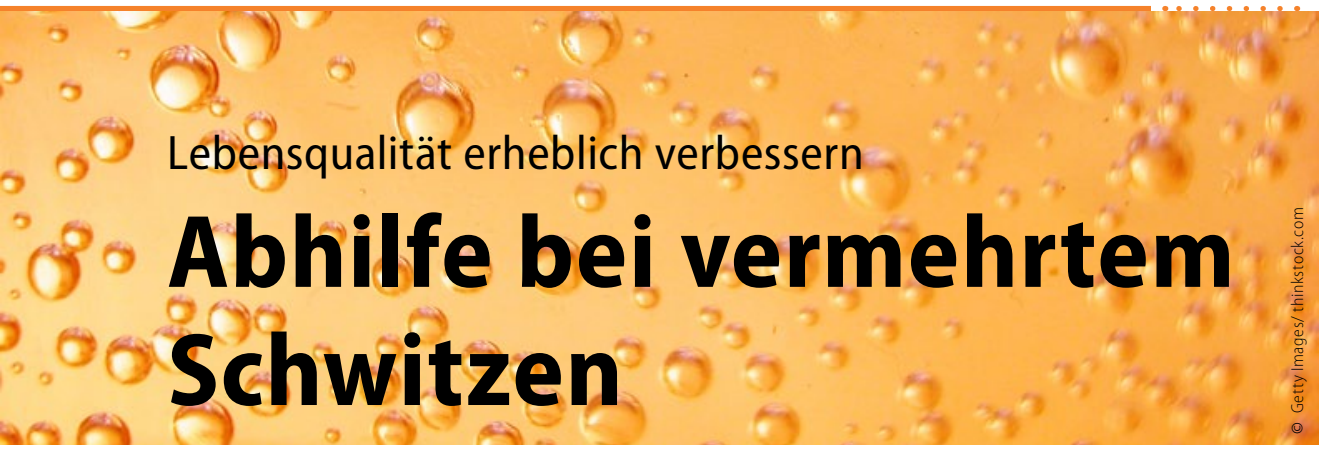

Hyperhidrose ist behandelbar.

Sichere Abhilfe schaffen u.a. orale

Anticholinergika, wie neue

Studienergebnisse zur Evidenz von

Methantheliniumbromid zeigen.

Insgesamt leiden 1-3\% der Bevölkerung unter fokaler Hyperhidrose, wobei am häufigsten Achseln, Hände und Füßen betroffen sind. Wichtiger Bestandteil des therapeutischen Arsenals sind orale Anticholinergika. Hierzu zählt auch Methantheliniumbromid (Vagantin ${ }^{\circledR}$ ), für das in dieser Indikation objektive und gut dokumentierte Daten vorliegen. Eine multizentrische, placebokontrollierte Studie mit insgesamt 339 Patienten bestätigte jetzt die Effektivität des Medikaments bei axillärer Hyperhidrose, erläuterte Prof. Dr. Berthold Rzany, Berlin. Binnen kurzer Zeit nach Tabletteneinnahme wurde die axil- läre Schweißmenge signifikant reduziert. Die Anzahl der Patienten, bei der die Schweißproduktion um mindestens $40 \%$ gemindert wurde, lag bei 77,3\%. Auch die Lebensqualität verbesserte sich über den Studienzeitraum von vier Wochen im Vergleich zum Ausgangswert erheblich. Da Methantheliniumbromid nicht ZNS-gängig ist, war im Vergleich zu anderen Anticholinergika auch nicht mit zentralnervös-bedingten Nebenerscheinungen wie Schwindel, Übelkeit oder Schlafstörungen zu rechnen. Die Substanz erwies sich folglich nicht nur als sicher, sondern auch als gut verträglich.

Pressegespräch "Nutzen der systemischen Hyperhidrosetherapie - ein Update" im Rahmen der "23. Fortbildungswoche für praktische Dermatologie und Venerologie", München, Veranstalter; Riemser Arzneimittel AG, Riems, Bericht: Yvette C. Zwick

\section{Zulassung für Vancomyin erweitert}

Ab sofort steht Vancomycin (als Enterocaps ${ }^{\circledast} 250 \mathrm{mg}$ ) von Riemser Arzneimittel auch zur Therapie von Patienten mit leichter und mittlerer Clostridium-difficile-assoziierter Diarrhoe (CDAD) zur Verfügung, teilt das Unternehmen mit. Das Bundesinstitut für Arzneimittel und Medizinprodukte habe einer Erweiterung der bisher zugelassenen Indikation von der schweren, pseudomembranösen Kolitis auf alle CDAD-Fälle zugestimmt. Die Leitlinien der European Society of Clinical Microbiology and Infectious Diseases (ESCMID) von 2009 sowie der Infectious Disease Society of America (IDSA), empfehlen orales Vancomycin wie etwa das genannte Präparat bereits als First-Line-Therapeutikum für schwere CDAD-Erkrankungen sowie für rezidivierende Erkrankungen. Aufgrund der Zulassungserweiterung könne das Präparat jetzt für die Behandlung aller CDAD-Patienten eingesetzt werden und ermögliche so jedem Patienten eine bedarfsgerechte Therapie - noch bevor sich eine schwere Erkrankung entwickelt.

Die CDAD ist die häufigste nosokomiale Durchfallerkrankung - die Inzidenz steigt jährlich um $30 \%$. Ein begünstigender Faktor für diesen Anstieg ist die soziodemografische Entwicklung.

In Deutschland leben immer mehr ältere Menschen mit immunschwächenden Begleiterkrankungen und Therapien, die den Ausbruch der CDAD begünstigen. Auch die steigende Anzahl von Krankenhausaufenthalten und die massive Anwendung von Antibiotika haben der Erkrankung Vorschub geleistet. Doch nicht nur in Kliniken, sondern vor allem im ambulanten Bereich und Pflegeheimen häufen sich die Krankheitsfälle. Durch die Kapselform mit monolithischem Wirkstoffkern sei Vancomycin Enterocaps ${ }^{\circledast} 250 \mathrm{mg}$ sehr gut zur Therapie ambulanter Patienten im häuslichen Umfeld und Pflegeheimen geeignet, so das Unternehmen.

(www.riemser.com) ,Sign Against Stroke in Atrial Fibrillation'", Veranstalter: Bayer Vital GmbH, Düsseldorf-Benrath 2012,

Bericht: Ludger Riem
Pressemitteilung von RIEMSER Arzneimittel $A G$

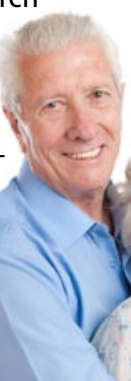

aller Regel eine konsequente Antikoagulation. Diesem Bedarf werden moderne
Pressekonferenz "Rathausgespräche: Dialog Schlaganfall - Vorstellung der weltweiten Patienten-Charta

Antikoagulantien wie der Faktor fenbar gerecht, so Prof. Dr. Erland ErdGeringere Inzidenz von Blutungen Rivaroxaban gegenuber einem VitaminK-Antagonisten als gleichermaßen effekell besser ab. Fatal verlaufende Blutungen traten signifikant seltener auf und die Inzidenz von Hirnblutungen war geringer.

Maßnahmen ließen sich nach Einschätung von Dr. Markus Wagner, Gütersloh, um die $70 \%$ aller Schlaganfälle vermeiden. 